\title{
Hyperuricemia, endothelial dysfunction and hypertension
}

\author{
Daniela MIRICESCU ${ }^{1}$, Alexandra TOTAN ${ }^{1}$, Constantin STEFANI ${ }^{2,3}$, lulia-loana STANESCU ${ }^{4}$, \\ Marina IMRE ${ }^{5}$, Ana Maria Alexandra STANESCU ${ }^{2}$, Radu RADULESCU ${ }^{1}$, Daniela BALAN ${ }^{4}$, \\ Andra-Elena BALCANGIU-STROESCU ${ }^{4,6}$, Maria GREABU ${ }^{1}$ \\ ${ }^{1}$ Department of Biochemistry, Faculty of Dental Medicine, "Carol Davila" University of Medicine and \\ Pharmacy, Bucharest, Romania \\ 2Department of Family Medicine, "Carol Davila" University of Medicine and Pharmacy, Bucharest, \\ Romania \\ 3"Carol Davila" University Central Emergency Military Hospital, Bucharest, Romania \\ "Department of Physiology, Faculty of Dental Medicine, "Carol Davila" University of Medicine and \\ Pharmacy, Bucharest, Romania \\ ${ }^{5}$ Department of Complete Denture, Faculty of Dental Medicine, "Carol Davila" University of Medicine \\ and Pharmacy, Bucharest, Romania \\ ${ }^{6}$ Department of Dialysis, Emergency University Hospital, Bucharest, Romania
}

\section{ABSTRACT}

The final product of purine bases degradation in humans, reptiles and other primates is uric acid. The serum level of uric acid can be influenced by diet, certain systemic pathologies and a decreased renal excretion. Xanthine oxidase (XO) catalyzes the formation of uric acid with generation of reactive oxygen species (ROS), being active in physiological stress and ischemia. Uric acid has an antioxidant role at the extracellular level, but also in certain biological fluids such as saliva. In the intracellular environment of endothelial cells it plays a prooxidant role in conditions of hyperuricemia, contributing to the decrease of nitric oxide (NO) level, leading to endothelial dysfunction and the development of hypertension. The purpose of this review is to present the synthesis and biological effects of uric acid, the effects of hyperuricemia on NO production, and the link between hyperuricemia, endothelial dysfunction and hypertension.
\end{abstract}

Keywords: uric acid, hyperuricemia, endothelial dysfunction, hypertension

\section{INTRODUCTION}

Purines play vital roles in the body - ATP (adenosine triphosphate) provides the energy needed to carry out intracellular reactions, while nitrogenous bases (adenine and guanine) are components of nucleic acids (DNA and RNA). The transformation of the mononu- cleotides AMP (adenosine monophosphate), GTP (guanidine monophosphate), ITP (inosine monophosphate) involves several enzymatic steps to obtain hypoxanthine and its further oxidation to xanthine. Oxidation of hypoxanthine to xanthine and xanthine to uric acid is accomplished by two isoforms of xanthine oxidoreductase, xanthine dehydrogenase (XDH) and 
$\mathrm{XO}$. The last isoform leads to the generation of ROS $\left(\mathrm{O}_{2}^{\cdot-}, \mathrm{H}_{2} \mathrm{O}_{2} \mathrm{O}_{2}^{\cdot-}, \mathrm{H}_{2} \mathrm{O}_{2}\right)$, being activated in physiological stress and ischemia. XO oxidase is an enzyme present in a high concentration in the intestine and liver, contains riboflavin, molybdenum and iron $(1,2)$.

\section{URIC ACID SYNTHESIS}

Uric acid is the final product of purine catabolism in humans, birds, reptiles and other primates, which don't contain the enzyme uricase. The serum uric acid level is influenced by diet, a diet rich in meat, fructose, alcohol, seafood and $\mathrm{Na}$, which will increase its concentration. On the other part, consumption of vitamin $\mathrm{C}$ and coffee decrease uric acid level (2).

The level of uric acid increases rapidly after fructose ingestion, fructokinase is activated, resulting in increments as high as $2 \mathrm{mg} / \mathrm{dl}$ within $1 \mathrm{~h}$ (3).

\section{BIOCHEMISTRY AND BIOLOGICAL ACTIONS OF URIC ACID}

Uric acid plays a dual role, antioxidant and pro-oxidant. In the hydrophilic extracellular environment, the uric acid is antioxidant, neutralizes peroxynitrile radicals $\left(\mathrm{ONOO}^{-}\right)_{\mathrm{ONOO}^{-}}$) and chelates metal ions (Fe, $\mathrm{Cu}$, II), thus preventing the formation of new ROS such as hydroxyl radical $\left(\mathrm{HO}^{\circ} \mathrm{HO}^{\circ}\right)$. By neutralizing $\mathrm{ONOO}^{-}$ $\mathrm{ONOO}^{-}$radicals by uric acid, the enzymatic activity of endothelial nitric oxide synthetase (eNOS) is stabilized (4). Uric acid as an antioxidant cannot neutralize the superoxide anion $\left.\left(\mathrm{O}_{2}^{*-}\right) \mathrm{O}_{2}^{*-}\right)$ and in the plasma needs vitamin $C$ to exercise its antioxidant role (5). Uric acid presents a preventive role in the development of neurodegenerative diseases such as dementia or bone metabolism by increasing bone mass and reducing bone turnover, involved in reducing vertebral fractures (6). In the oral cavity, uric acid is the most important antioxidant, achieving $85 \%$ of the total antioxidant capacity. Salivary uric acid levels are decreased in oral pathologies associated with oxidative stress (OS), such as periodontal disease, oral lichen planus and oral cancer (7-10). Unfortunately in the hydrophobic intracellular environment, it plays a prooxidant role. The XO enzyme catalyzes the last two stages of purine catabo- lism generating $\mathrm{O}_{2}^{--}$and $\mathrm{H}_{2} \mathrm{O}_{2} \mathrm{O}_{2}^{\bullet-}$ and $\mathrm{H}_{2} \mathrm{O}_{2}$ (11-12). The intracellular prooxidant role of uric acid is mediated by a NADPH oxidase-dependent pathway. In several systemic pathologies (diabetes, cardiovascular diseases, ischemic liver injury) plasma uric acid is a marker of oxidant damage (13-15).

\section{HYPERURICEMIA}

Hyperuricemia occurs when uric acid levels exceed $339 \mu \mathrm{mols} / \mathrm{l}$ for premenopausal women and 416 umols/l for men and postmenopausal women, and urate crystals will be deposited in organs and tissues (16). This difference between the sexes is due to the effect of female sex hormones (17). At a concentration higher than $10 \mathrm{mg} / \mathrm{dl}$ (600 $\mu$ mols/l) uric acid will be deposited in the joints and soft tissues. Uric acid can be oxidized to allantoin - a much more soluble compound, by uricase (urate oxidase). Because of two truncating mutations in the uricase gene, which introduce premature stop codon, the human species no longer synthesizes the uricase gene (18). Uric acid is a paradoxical biomolecule, in the intracellular environment of vascular smooth muscle cells (VSMC) and adipocytes play prooxidant role. The negative effects of uric acid are manifested by decreased NO production, induces platelet aggregation and pro-inflammatory activity (19).

Hyperuricemia is associated with many comorbidities, such as cardiovascular diseases, diabetes, dyslipidemia, metabolic syndrome, chronic kidney disease and obesity. Hypertension, atrial fibrillation, coronary atherosclerotic heart disease, heart failure are cardiovascular conditions associated with hyperuricemia $(15,20)$.

Numerous factors can produce an increase of purines or uric acid synthesis, such as: clinical conditions (obesity and insulin resistance; cell turnover - haematological disorders, malignant processes, severe proliferative psoriasis; hypoxia, ischemic tissue; Down Syndrome), genetic disorders that lead to metabolic errors associated with enzymatic deficiencies (phosphoribosyl transferase deficiency; hyperactivity for phosphoribosylphosphate synthetase; glucozo-6-phosphatase), diet (excessive ingestion of purines, alcohol, fructose,

$$
\begin{gathered}
\text { Fructose }+A T P \stackrel{(F K / K H K)}{\longrightarrow} \text { Fructose }-1-P+A D P \\
A D P \stackrel{\text { Adenylate kinase }}{\longrightarrow} A M P \stackrel{A M D P 2}{\longrightarrow} I M P \stackrel{S I N T}{\longrightarrow} \text { Inosine } \rightarrow \text { Hypoxanthine } \\
\text { Hypoxanthine } \stackrel{X Q}{\longrightarrow} \text { Xanthine }+\mathrm{H}_{2} \mathrm{O}_{2} \stackrel{X O}{\longrightarrow} \text { Uric acid }+\mathrm{H}_{2} \mathrm{O}_{2}
\end{gathered}
$$

FIGURE 1. The mechanism by which fructose from the diet contributes to uric acid synthesis - adapted from (2)

FK - fructokinase; KHK - ketohexokinase; AMPD2 - AMP deaminase 2; 5'NT - 5' nucleotidase 
deficient B12, niacin), drugs or toxins (cytotoxic agents).

Moreover, urinary secretion can be decreased by several clinical conditions (renal failure, obesity, lactic acidosis, hyperparathyroidism, hypothyroidism, heart damage), genetic disorders (genetic polymorphism of genes that encodes urate transporters: GLUT9, URAT1), diet, alcohol, dehydration and ketoacidosis starvation, or by drugs or toxins (beta blockers, diuretics, laxative abuse, beryllium, calcineurin inhibitors, levodopa, low-dose salicylates, pyrazinamide, methoxyflurane) (21).

\section{HYPERURICEMIA AND NO}

Several mechanisms have been described so far by which hyperuricemia leads to decreased NO production in endothelial cells.

The increased uric acid level determines the decrease of NO production by generating ROS, with a central role belonging to $\mathrm{XO}$. Studies on human umbilical endothelial cell lines conducted by Li et al., reported that hyperuricemia induces ROS formation and PKC (protein kinase C) signaling pathway activation and phosphorylation of Threonine 495 (Thr) from eNOS structure (22). Thr phosphorylation reduces the activity of the enzyme, Serine (Ser) phosphorylation, increases eNOS activity (22). eNOS phosphorylation at Thr due to OS reduces the interaction between enzyme and calmodulin and decreased NO production (23).

The second mechanism by which hyperuricemia reduces NO production is through two signaling pathways in which the insulin receptor is involved. Insulin binds to its specific receptor, leading to PI3K (phosphoinositol 3 kinase) or AkT (protein kinase B) signaling pathway activation, which promotes metabolic effects (cell growth and survival, glycogen metabolism). MAPK (mitogen-activated protein kinase) is also activated being involved in cell proliferation and differentiation, and gene expression. Insulin stimulates eNOS phosphorylation at Ser 1147, enhancing enzyme activity, but uric acid blocks this phosphorylation reaction (24). Elevated uric acid levels cause recruitment of the enzyme EN-PP1 (endonucleotide pyrophosphate/ phosphodiesterase 1) at plasma membrane, of all insulin targeted tissues, and inhibit insulin receptor, affecting eNOS phosphorylation via PI3K/Akt signaling pathway (25).

In addition, uric acid stimulates through insulin receptor the gene expression for endothelin 1 (ET-1), the most important vasoconstrictor molecule, through MAPK / ERK (extracellular signal-regulated kinase) signaling pathways (26). Hyperuricemia inhibits vasodilation and stimulates vasoconstriction via insulin (25-27).
Also, NO is synthesized in endothelial cells by eNOS from L-arginine, which reaches the endothelial level via transmembrane transporter CAT (cation amino acid transporter). In pulmonary arterial endothelial cells, studies have shown that elevated uric acid levels enhance arginase activity, by attenuating cyclic GMP production, leading to arginine catabolism (23). Hyperuricemia reduces the uptake of arginine into endothelial cells (28).

Moreover, hyperuricemia enhanced activities for renin and angiotensin system (RAS). These findings were reported by clinical trials using laboratory animals (rabbits) (29). NO together with the RAS system maintain the normal function of blood vessels. NO relaxes VSMC, while RAS has vasoconstrictor action. Excess uric acid reacts with NO, leading to the formation of an unstable compound (nitrosouric acid), with 6 aminouracil formation in the end. 6 aminouracil will diminish NO role and enhance RAS activity in blood vessels (30). The renal epithelial sodium channel $(\mathrm{ENaC})$ plays an important role in maintaining the $\mathrm{Na}$ balance, extracellular fluid volume, blood pressure, being responsible for the stage of limiting the rate of $\mathrm{Na}$ reabsorption. $\mathrm{ENaC}$ can be activated in conditions of hyperuricemia (31). Studies in rats with hyperuricemia, reported that $\alpha-, \beta$ - and $\gamma$-ENaC expressions were significantly increased, enhancing sodium ions absorption in the renal tubules and leading to increased blood volume and in the end to hypertension (31).

\section{HYPERURICEMIA AND ENDOTHELIAL DYSFUNCTION}

Endothelial dysfunction together with vascular stiffness are involved in the progression and development of cardiovascular and cardiorenal diseases. The mechanisms by which uric acid promotes cardiovascular damage are multiple, involving the existence of OS, reduced bioavailability of $\mathrm{NO}$, inflammatory response and maladaptive cardiac, vascular, renal hepatic, and adipocyte immunity (3). There are two mechanisms by which hyperuricemia causes arterial stiffness: urate crystal mechanism and crystal-independent mechanism.

$$
\begin{gathered}
\text { Hyperuricemia and uric acid crystal formation } \\
\downarrow \\
\text { Macrophages engulf cristal } \\
\downarrow \\
\text { NLRP3 inflammasome activation } \\
\downarrow \\
I L-1 \beta \text { production } \\
\downarrow \\
\text { Inflammation and collagen production } \\
\downarrow \\
\text { Proliferation of smooth muscle }
\end{gathered}
$$

FIGURE 2. Urate crystal mechanism (adapted from 32). NLRP3 -nod-like receptor family protein 


\author{
Hyperuricemia \\ $\downarrow$ \\ Intracellular and mitochondrial OS \\ $\downarrow$ \\ eNos inhibation \\ $\downarrow$ \\ GLU9 (URATEv1) transport urate into vessels \\ $\downarrow$ \\ RAS stimulation \\ $\downarrow$ \\ Inflammation \\ $\downarrow$ \\ Vasocontraction \\ $\downarrow$ \\ Endothelium dysfunction \\ $\downarrow$ \\ Smooth muscle proliferation + endothelium \\ dysfunction \\ $\downarrow$ \\ Arterial stiffness and Arteriosclerosis \\ $\downarrow$ \\ Hypertension
}

FIGURE 3. The independent-crystal mechanism ladapted from 32). URATV1 - voltage-driven urate efflux transporter 1

At endothelial level, uric acid crystals deposited, together with subendothelial lipids, will lead to vascular endothelial damage, inducing further vascular endothelial injury and finally atherosclerosis. Damage of endothelial cells will weaken their anticoagulant role, subendothelial collagen will be exposed to injury and will induce platelet aggregation and adhesion, promoting thrombus formation (33).

\section{HYPERURICEMIA AND HYPERTENSION}

According to cohort, cross-sectional and interventional studies conducted so far, hyperuricemia is an independent risk factor in hypertension. Hyperuricemia is present in hypertensive patients who are obese, diabetic, or have dyslipidemia, being involved in the production of atheroma plaque. Clinical studies have reported the association between hyperuricemia and atherosclerosis, with the presence of coronary artery calcifications, the decreased flow-mediated dilation, which indicates the vascular endothelial function and higher pulse wave velocity (32-36).

Hyperuricemia along with microalbuminuria is associated with prehypertensive patients. Uric acid levels greater than $5.5 \mathrm{mg} / \mathrm{dl}$ were observed in $90 \%$ of adolescents with essential hypertension, shared with healthy subjects and adolescents with secondary hypertension. Hyperuricemia was observed in $40-60 \%$ of untreated hypertensive patients $(35,36)$. Animal studies have reported the association of hypertension with elevated uric acid levels that mediate renal vasoconstriction by reducing endothelial NO levels and activating RAS. Rats with hyperuricemia over time will develop essential hypertension and renal microvascular damage (37-39).

\section{CONCLUSIONS}

Uric acid is a biomolecule with a duplicative role that has an antioxidant role in the extracellular environment and prooxidant in the intracellular medium. Several clinical pathologies, genetics, diet, but also a decreased urinary excretion lead to hyperuricemia. Hyperuricemia conduce to decreased NO level, by involving OS and activating PKC signaling pathway, which will lead to decreased activity of the eNOS, involved in NO production from L-arginase. Increased serum uric acid levels reduce the entry of the amino acid arginine into endothelial cells, activates the RAS system, promoting endothelial dysfunction and hypertension. Decreasing uric acid levels will have beneficial effects on patient's health.

\section{Acknowledgement}

All authors equally contributed to the present paper.

Conflict of interest: none declared Financial support: none declared

\section{REFERENCES}

1. Zou H, Wang H, Liu T et al. Protective role of a-lipoic acid in hyperuricemia induced endothelial dysfunction. Exp Ther Med 2017;13:3047-3054.

2. Johnson JR, Nakagawa T, Sanchez-Lozada $G$ et al. Sugar, Uric Acid, and the Etiology of Diabetes and Obesity. Diabetes 2013;62:3307-3315.

3. Kunal C, Kunal M, James S et al. Uric Acid - key ingredient in the recipe for Cardiorenal Metabolic Syndrome. Cardiorenal Med 2013;3(3): 208-220.
4. Nuzkaya N, Weissmann N, Harrison DG et al. Interactions of peroxynitrite with uric acid in the presence of ascorbate and thiols: implications for uncoupling endothelial nitric oxide synthase. Biochem Pharmacol 2005;70(3):343-354.

5. Stephen $P$, Juraschek E, Miller R et al. Effect of oral vitamin $C$ supplementation on serum uric acid: a meta-analysis of randomized controlled trials. Arthritis Care Res 2011;63(9):1295-1306.
6. Muka T, de Jonge EA, Kiefte-de Jong JC et al. The influence of serum uric acid on bone mineral density, hip geometry, and fracture risk: the rotterdam study. J Clin Endocrinol Metab 2016;101:1113-1122.

7. Miricescu D, Totan A, Calenic B et al. M Salivary biomarkers: Relationship between oxidative stress and alveolar bone loss in chronic periodontitis. Acta Odontol Scand 2014;72(1):42-7.

8. A Totan, Miricescu D, Parlatescu I et al. New possible salivary and serum 
biomarkers in oral lichen planus. Biotechnic and Histochemistry 2015;19:1-7.

9. Miricescu D, Greabu M, Totan A et al. The antioxidant potential of saliva: clinical significance in oral diseases. Therapeutics, Pharmacology and Clinical Toxicology 2011;15(2):139-143.

10. Totan A, Greabu M, Stanescu II et al. Uric acid - history, present and future. Revista Medicală Română 2019;66(2):150-152.

11. Kun-Mo L, Chien-Lin L, Kuo-Chin $\mathrm{H}$ et al. The paradoxical role of uric acid in osteoporosis. Nutrients 2019;11:2111.

12. Muiesan ML, Agabiti-Rosei C, Paini A et al. Uric Acid and Cardiovascular Disease: An Update Eur Cardiol 2016; 11(1): 54-59.

13. Ruggiero CA, Herubini A. Ble A et al. Uric acid and inflammatory markers. European Heart J 2006;27(10):1174-1181.

14. Lyngdoh T, Marques-Vidal P, Paccaud F et al. Elevated serum uric acid is associated with high circulating inflammatory cytokines in the population-based colaus study. PLOS One 2011;6 (5):e19901.

15. Treviño-Becerra A, Iseki K. Uric Acid in Chronic Kidney Disease. Contrib Nephrol Basel Karger 2018;192:82-87.

16. Zou H, Wang H, Liu T et al. Protective role of $a$-lipoic acid in hyperuricemia induced endothelial dysfunction. Exp Ther Med 2017;13:3047-3054.

17. Tuven $B$, Soysal $P$, Unutmaz $G$ et al. Uric acid may be protective against cognitive impairment in older adults, but only in those without cardiovascular risk factors. Exp Gerontol 2017;89:15-19.

18. Wu XW, Lee CC, Muzny DM et al. Urate oxidase: primary structure and evolutionary implications. Proc Natl Acad Sci U S A 1989;86(23):9412-9416.

19. Choi H, Kim HC, Song BM et al. Serum uric acid concentration and metabolic syndrome among elderly Koreans: the Korean urban rural elderly (KURE) study. Arch Gerontol Geriatr 2016; 64:51-58.
20. Kuwabara, M. Hyperuricemia, cardiovascular disease, and hypertension. Pulse (Basel). 2016;3:242-252.

21. Stewart DJ, Langlois V, Noone D. Hyperuricemia and Hypertension: Links and Risks. Integr Blood Press Control 2019;12:43-62.

22. Li P, Zhang L, Zhang M et al. Uric acid enhances PKC-dependent eNOS phosphorylation and mediates cellular ER stress: a mechanism for uric acid-induced endothelial dysfunction. Int J Mol Med 2016;37:989-997.

23. Haque MM, Ray SS, Stuehr DJ. Phosphorylation controls endothelial nitric-oxide synthase by regulating its conformational dynamics. J Biol Chem 2016;291:23047-23057.

24. Tassone EJ, Cimellaro A, Perticone M et al. Uric acid impairs insulin signaling by promoting Enpp1 binding to insulin receptor in human umbilical vein endothelial cells. Front Endocrinol (Lausanne) 2018; 9: 98.

25. Choi YJ, Yoon Y, Lee KY et al. Uric acid induces endothelial dysfunction by vascular insulin resistance associated with the impairment of nitric oxide synthesis. FASEB J 2014;28:3197-3204.

26. Dubó S, Gallegos D, Cabrera L et al. Cardiovascular action of insulin in health and disease: endothelial l-arginine transport and cardiac voltage-dependent potassium channels. Front Physiol 2016;7:74.

27. Turaihi AH, Bakker W, van Hinsbergh VW et al. Insulin receptor substrate 2 controls insulin-mediated vasoreactivity and perivascular adipose tissue function in muscle. Front Physiol 2018;9:245.

28. Schwartz IF, Grupper A, Chernichovski T et al. Hyperuricemia attenuates aortic nitric oxide generation, through inhibition of arginine transport, in rats. $J$ Vasc Res 2011;48:252-260.

29. Zheng H, Li N, Ding Y et al. Losartan alleviates hyperuricemia-induced atherosclerosis in a rabbit model. Int $\mathrm{J}$ Clin Exp Pathol 2015;8:10428-1035.

30. Chen C, Lü JM, Yao Q. Hyperuricemiarelated diseases and xanthine oxidoreductase (XOR) inhibitors: An overview. Med Sci Monit 2016;22:25012512.

31. Xu W, Huang Y, Li L et al. Hyperuricemia induces hypertension through activation of renal epithelial sodium channel (ENaC). Metabolism 2016;65:73-83.

32. Masanari K, Mehmet K, Ichiro H. Uric Acid and Hypertension Because of Arterial Stiffness. Hypertension 2018;72(3):582584.

33. Srikanthan K, Feyh A, Visweshwar H et al. Systematic review of metabolic syndrome biomarkers: A panel for early detection, management, and risk stratification in the West Virginian population. Int J Med Sci 2016;13:25-38.

34. Feig DI. Hyperuricemia and Hypertension. Adv Chronic Kidney Dis. 2012;19 (6):377-385.

35. Masanari K. Hyperuricemia, Cardiovascular Disease, and Hypertension. Pulse (Basel) 2016; 3(3-4):242-252.

36. Yi-Bang C, Yan L. Does It Matter for the Progression From Prehypertension to Hypertension?. Hypertension 2018;71(1):66-67.

37. Feig DI, Kang DH, Johnson RJ. Uric acid and cardiovascular risk. N Engl J Med. 2008;359(17):1811-21.

38. Fan H, Aijuan Z, Pengfei X et al. HighProtein Diet Induces Hyperuricemia in a New Animal Model for Studying Human Gout. Int J Mol Sci 2020;21(6):2147.

39. Yantong Z, Xiaofei P, Guanghui L. An Update on the Animal Models in Hyperuricaemia Research. Clin Exp Rheumatol 2017;35(5):860-864. 